\title{
Risk factors for illness severity in patients with COVID-19 pneumonia: a prospective cohort study
}

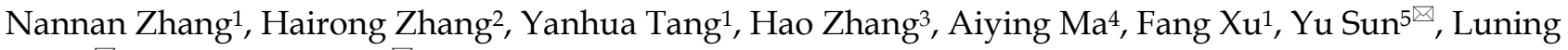 \\ Jiang ${ }^{1 \bowtie}$, Fangzhen Shan ${ }^{6,7 凶}$ \\ 1. Department of Pulmonary and Critical Care Medicine, Affiliated Hospital of Jining Medical University, Jining 272029, Shandong, China. \\ 2. Department of surgery, Jining Infectious Disease Hospital, Jining City 272000, Shandong, China. \\ 3. Department of hematology, Affiliated Hospital of Jining Medical University, Jining 272029, Shandong, China. \\ 4. Department of Intensive care unit II, Affiliated Hospital of Jining Medical University, Jining 272029, Shandong, China. \\ 5. Department of infectious disease, Jining Infectious Disease Hospital, Jining City 272000, Shandong, China. \\ 6. Medical Research Center, Affiliated Hospital of Jining Medical University, Jining 272029, Shandong, China. \\ 7. Hefei National Laboratory for Physical Science at Microscale and School of Life Science, University of Science and Technology of China, Hefei 230026,
} Anhui, China.

$\triangle$ Corresponding authors: Yu Sun, Tel.: +86-0537-290-4823; Email: 872543883@qq.com. Luning Jiang, Tel.: +86-0537-290-3971; Email: w13001786181@126.com. Fangzhen Shan, Tel.: +86-0537-290-3579; Email: fzshan@mail.ustc.edu.cn.

(C) The author(s). This is an open access article distributed under the terms of the Creative Commons Attribution License (https://creativecommons.org/licenses/by/4.0/). See http://ivyspring.com/terms for full terms and conditions.

Received: 2020.07.28; Accepted: 2020.12.04; Published: 2021.01.01

\begin{abstract}
Background: Although COVID-19 pneumonia is spreading internationally, knowledge regarding the factors associated with the illness severity of patients remains limited. We aimed to identify the factors associated with the disease severity of patients with COVID-19 pneumonia induced by a novel coronavirus, severe acute respiratory syndrome coronavirus 2 (SARS-CoV-2).

Methods: We prospectively enrolled a single-center case series of adult patients with COVID-19 admitted to the Infectious Disease Hospital of Jining, Jining City, Shandong Province, China, from January 24 to March 1, 2020. Demographics, clinical characteristics, and laboratory findings were compared to investigate the risk factors related with the disease severity of COVID-19 pneumonia patients.

Results: We included a total of 78 patients with COVID-19 pneumonia, of whom 6 had the severe type. As compared to a moderately ill cohort, our analysis showed that shortness of breath, fatigue, longer days from illness onset to diagnosis confirmed, neutrophil percentages $>70 \%$, neutrophil counts $>6.3 \times 10^{9} / \mathrm{L}$, lymphocyte percentages $<20 \%$, lymphocyte counts $<1.0 \times 10 \%$ L, platelet $<100 \times 10 \% / \mathrm{L}$, C-reactive protein $(\mathrm{CRP})>10 \mathrm{mg} / \mathrm{L}$, neutrophil to platelet ratio $(\mathrm{NPR})>2.3$, neutrophil to lymphocyte ratio (NLR) $>3.9$, aspartate aminotransferase (AST) $>40 \mathrm{U} / \mathrm{L}$, albumin $<40 \mathrm{~g} / \mathrm{L}$, lactate dehydrogenase $(\mathrm{LDH})>245$ $\mathrm{U} / \mathrm{L}$, and glucose $>6.1 \mathrm{mmol} / \mathrm{L}$ were predictors of disease severity in COVID-19 pneumonia. In the sex-, age-, and comorbid illness-matched case-control study, neutrophil percentages $>70 \%$, neutrophil counts $>6.3 \times 10 \% / \mathrm{L}$, lymphocyte percentages < 20\%, NPR > 2.3, NLR > 3.9, albumin < $40 \mathrm{~g} / \mathrm{L}$, and LDH > $245 \mathrm{U} / \mathrm{L}$ remained associated with the early detection and identification of severe patients.

Conclusion: We demonstrated that neutrophil percentages $>70 \%$, neutrophil counts $>6.3 \times 10 \% / \mathrm{L}$, lymphocyte percentages < 20\%, NPR > 2.3, NLR > 3.9, albumin $<40 \mathrm{~g} / \mathrm{L}$, and LDH $>245 \mathrm{U} / \mathrm{L} \mathrm{might}$ predict the severity of illness in patients with COVID-19 pneumonia.
\end{abstract}

Key words: SARS-CoV-2; COVID-19; severity; risk factor; prospectivestudies

\section{Background}

COVID-19 pneumonia has become a worldwide pandemic since it first emerged in December, 2019, in Wuhan City, Hubei Province, China [1-3]. The severe acute respiratory syndrome coronavirus 2 (SARS-CoV-2), belonging to the $\beta$-coronavirus family, is similar to some known coronaviruses, such as SARS-CoV and middle east respiratory syndrome (MERS)-CoV, in terms of its surface spike proteins [4, 5]. As at September 15, 2020, the COVID-19 pneumonia pandemic has been reported in 188 
countries globally, with 29,316,738 confirmed cases and 929,236 deaths [6]. COVID-19 has been declared a public health emergency by the World Health Organization, threatening global health and economies. Unfortunately, the COVID-19 pneumonia pandemic is still ongoing and not well controlled at present.

Emerging studies have reported the epidemiology and clinical characteristics of COVID-19. Symptoms of SARS-CoV-2 infection range from none to critical illness, and often present as fatal acute respiratory distress syndrome [7-9]. In one published study including 99 patients with COVID-19 pneumonia from Wuhan Jinyintan Hospital, 17\% patients developed severe symptoms [10]. The percentage of severe cases was $11.3 \%$ in another cohort of hospitalized patients with COVID-19 pneumonia [11]. Moreover, in a study in the New York City Area from America, a case series involved 5700 patients, the percentage of severe cases were $14.2 \%$ [12]. Though results from these three studies have indicated that older age, and multiple pulmonary lobes involvement and pleural effusion were associated with the illness severity, factors related with the severity of illness are still poorly understood [13]. Initial studies were mostly retrospectively; whereas, prospective studies on the prediction of disease severity are limited [14-17].

In the present study, a total of 78 adult patients confirmed with COVID-19 infection hospitalized at the Institute of Infectious Diseases, Jining Hospital, which was a COVID-19-designated hospital, between January 24 and March 1, 2020 were recruited. The patients included 72 moderate and 6 severe cases, on the basis of The Diagnosis and Treatment of COVID-19 guidelines (7th version) published by the National Health Commission of China [18]. We compared the differences in clinical characteristics and laboratory findings between moderate and severe cases, in order to identify the clinical and laboratory parameters related to illness severity of patients with COVID-19 pneumonia. It was identified that if neutrophil percentages $>70 \%$, neutrophil counts $>6.3$ $\times 10^{9} / \mathrm{L}$, lymphocyte percentages $<20 \%$, NPR $>2.3$, NLR > 3.9, albumin < $40 \mathrm{~g} / \mathrm{L}$, and LDH $>245 \mathrm{U} / \mathrm{L}$ might be risk factors for disease severity among patients with COVID-19 pneumonia. Our study will provide a supplement to the researches on epidemic of COVID-19 pneumonia, and references for its diagnosis.

\section{Methods}

\section{Study patients and data collection}

Clinical characteristics and laboratory findings were prospectively collected from a single-center cohort of 78 consecutive adult patients confirmed with COVID-19 pneumonia who were admitted to Jining Infectious Disease Hospital, Jining City, Shandong Province, China, from southeast Shandong province, between January 24 and March 1, 2020. All patients were diagnosed with COVID-19 through a real-time reverse transcriptase-polymerase chain reaction assay using oropharyngeal swab specimens from the upper respiratory tract. We divided the disease severity into a moderate or severe group based on the latest Chinese COVID-19 Management Guidance (7th edition) [18]. Our study was approved by the Ethics Committee of the Jining Infectious Disease Hospital, Jining City, Shandong Province, China (No. 20200102). All methods were performed in accordance with the relevant guidelines and regulations. Each patient provided informed consent at the time we started to collect and analyze the data.

Medical information of each patient including demographic data, clinical characteristics, laboratory results, and outcomes was extracted prospectively through review of electronic medical records. All the data were checked and reviewed by two doctors from an affiliated Hospital of Jining Medical University, independently.

\section{Statistics}

All statistical tests were performed using SPSS version 23.0 (SPSS Inc., Chicago, IL, USA). Continuous variables are presented as means \pm standard deviations (SD) or median with interquartile range (IQR), and were compared using either the Student's t-test for normally distributed variables or the Mann-Whitney test for non-normally distributed variables, as appropriate. Continuous variables were tested for normality using the Shapiro-Wilk test. Differences between categorical variables were determined using Pearson's Chi-squared test or Fisher's Exact Test, as appropriate, and presented as frequencies and percentages (\%). Statistical analyses were two-sided and significance was set at $P<0.05$.

\section{Results}

\section{Clinical characteristics on admission}

A cohort of 78 patients who were hospitalized at Jining Infectious Disease Hospital, Jining City, Shandong Province, China, between January 24 and March 1, 2020 were recruited. All patients were diagnosed with COVID-19 pneumonia by a positive SARS-CoV-2 nucleic acid test. As shown in Table 1, the median age of patients was $43.82 \pm 15.91$ years, and males accounted for $76.9 \%$. Of 78 patients, 6 $(7.7 \%)$ cases developed into a severe type. As for comorbidities, there were no significant differences in the percentages of hypertension, diabetes, 
cardiovascular disease, tumor, cerebrovascular disease, and lung disease between the moderate and severe cohorts (all $P>0.05$ ). We surveyed smoking habits and alcohol consumption in all patients. No significant differences were found in term of the sex percentage, coexisting disorders, smoking, or alcohol consumption between these two groups (all $P>0.05$ ).

Among the clinical symptoms including fever, shortness of breath, dry cough, fatigue, sputum production, gastrointestinal symptoms, hemoptysis, myalgia, headache, pharyngodynia, and rhinobyon, the top five common symptoms were fever $(80.8 \%)$, followed by dry cough (46.2\%), gastrointestinal symptoms $(17.9 \%)$, fatigue $(14.1 \%)$, and shortness of breath $(10.3 \%)$ in the total patient population. The mean age of the severe group was not different from that of the moderate cohort. We noted that shortness of breath and fatigue appeared more frequently in the severe cohort than in the moderate cohort $(50 \%$ vs. $6.9 \%, P=0.013 ; 50 \%$ vs. $11.1 \%, P=0.034)$. Furthermore, the days from illness onset to diagnosis confirmed in severe group were significantly longer than that in moderate group [9.0 (6.0-16.0 vs. 5.0 (3.0-7.0), $P=0.042]$.

\section{Laboratory data}

As compared to patients with moderate disease, the severe patients had a higher level of white blood cells (WBC) [8.35 (5.73-13.15) vs. $5.16(4.15-6.46) \times$ $\left.10^{9} / \mathrm{L}, P=0.008\right]$, neutrophil percentages $[86.95$ (76.9-91.88)\% vs. 55.2 (48.08-65.35)\%, $P<0.001]$, neutrophil counts [6.47 (5.1-11.09) vs. 2.84 (2.08-3.88) $\left.\times 10^{9} / \mathrm{L}, P=0.001\right]$, and C-reactive protein (CRP) [58.08 (19.26-90.37) vs. $0.54(0.50-4.06) \mathrm{mg} / \mathrm{L}, P<$ 0.001 ], but lower levels of lymphocyte percentages [9.0 (4.08-13.38)\% vs. $31.65(22.6-39.95) \%, P<0.001]$, lymphocyte counts [0.74 (0.42-1.31) vs. 1.57 (1.18-1.89) $\left.\times 10^{9} / \mathrm{L}, P=0.002\right]$, and platelets [185 (79-230.75) vs. $\left.243.75(195.0-308.75) \times 10^{9} / \mathrm{L}, P=0.022\right]$ (Table 2). According to the parameters from the peripheral blood routine tests, we then calculated the neutrophil to platelet ratio (NPR), neutrophil to lymphocyte ratio (NLR), and platelet to lymphocyte ratio (PLR), in order to further investigate the factors related with illness severity between the mild and severe groups. Notably, indices including NPR [4.64 (2.40-11.44) vs. 1.24 (0.88-1.67), $P<0.001]$ and NLR [9.79 (5.87-22.96) vs. $1.72(1.17-2.77), P<0.001]$ were significantly increased in severe patients as compared to those in moderate cases.

Compared to the patients in the moderate group, severe patients were more susceptible to hepatic insufficiency, as indicated by the elevation of aspartate aminotransferase (AST) [33.5 (23.75-68) vs. $21(18-27) \mathrm{U} / \mathrm{L}, P=0.004]$ and lactate dehydrogenase
(LDH) [389.5 (241.5-497.5) vs. 197.5 (160.25-229.75) $\mathrm{U} / \mathrm{L}, \quad P=0.002]$, and the decreased albumin concentration [35.5 (32.75-38.25) vs. 42 (39-44) g/ L, $P=$ $0.003]$. In addition, severe patients also showed more frequently disturbed blood electrolytes and impaired glucose, as demonstrated by the reduction of sodium [136 (134.25-137.5) vs. 141 (139-143) $\mathrm{mmol} / \mathrm{L}, P=$ $0.001]$ and calcium [96.5 (93.75-98) vs. 100 (97-102) $\mathrm{mmol} / \mathrm{L}, P=0.015]$, and the increased glucose level [6.2 (5.63-11.5) vs. $4.9(4.4-5.6) \mathrm{mmol} / \mathrm{L}, P=0.007]$ (Table 2).

\section{Factors associated with disease severity}

For all the demographic data, clinical characteristics, and laboratory data, our analyses revealed that the severe group had longer days from illness onset to diagnosis confirmed, and significant higher proportions of patients showing shortness of breath, fatigue, neutrophil percentages $>70 \%$, neutrophil counts $>6.3 \times 10^{9} / \mathrm{L}$, lymphocyte percentages $<20 \%$, lymphocyte counts $<1.0 \times 10^{9} / \mathrm{L}$, platelet $<100 \times 10^{9} / \mathrm{L}, \mathrm{CRP}>10 \mathrm{mg} / \mathrm{L}, \mathrm{NPR}>2.3$, NLR $>3.9$, AST $>40 \mathrm{U} / \mathrm{L}$, albumin $<40 \mathrm{~g} / \mathrm{L}, \mathrm{LDH}>245$ $\mathrm{U} / \mathrm{L}$, and glucose $>6.1 \mathrm{mmol} / \mathrm{L}$ than that the moderate cohort (Table 1, Table 2). Therefore, our findings suggest that these factors might be associated with the disease severity of patients with COVID-19 pneumonia.

Table 1. Summary of patient clinical characteristics.

\begin{tabular}{|c|c|c|c|c|}
\hline Characteristics & $\begin{array}{l}\text { Total }(\mathrm{n}= \\
78)\end{array}$ & $\begin{array}{l}\text { Moderate } \\
(\mathrm{n}=72)\end{array}$ & $\begin{array}{l}\text { Severe }(n= \\
6)\end{array}$ & $\begin{array}{l}P \\
\text { value }\end{array}$ \\
\hline Age (years) & $43.82 \pm 15.91$ & $43.47 \pm 16.00$ & $48.00 \pm 15.49$ & 0.507 \\
\hline Gender, male, n (\%) & $60(76.92)$ & $45(62.50)$ & $5(83.33)$ & 0.562 \\
\hline \multicolumn{5}{|l|}{ Coexisting diseases, $\mathrm{n}(\%)$} \\
\hline Hypertension & $11(14.10)$ & $10(13.89)$ & $1(16.67)$ & 1.000 \\
\hline Diabetes & $11(14.10)$ & $10(13.89)$ & $1(16.67)$ & 1.000 \\
\hline Cardiovascular disease & $8(10.26)$ & $8(11.11)$ & 0 & 1.000 \\
\hline Tumor & $2(2.56)$ & $2(2.78)$ & 0 & 1.000 \\
\hline Cerebrovascular disease & $3(3.85)$ & $3(4.17)$ & 0 & 1.000 \\
\hline Smoking, n (\%) & $7(8.97)$ & $6(8.33)$ & $1(16.67)$ & 0.442 \\
\hline Alcohol consumption, n (\%) & $8(10.26)$ & $7(9.72)$ & $1(16.67)$ & 0.490 \\
\hline \multicolumn{5}{|l|}{ Symptoms, n (\%) } \\
\hline Fever & $63(80.77)$ & $57(79.17)$ & $6(100.00)$ & 0.481 \\
\hline Shortness of breath & $8(10.26)$ & $5(6.94)$ & $3(50.00)$ & 0.013 \\
\hline Dry Cough & $36(46.15)$ & $33(45.83)$ & $3(50.00)$ & 1.000 \\
\hline Fatigue & $11(14.10)$ & $8(11.11)$ & $3(50.00)$ & 0.034 \\
\hline Sputum production & $6(7.69)$ & $6(8.33)$ & 0 & 1.000 \\
\hline Gastrointestinal symptoms & $14(17.95)$ & $12(16.67)$ & $2(33.33)$ & 0.639 \\
\hline Hemoptysis & $1(1.28)$ & 0 & $1(16.67)$ & 0.077 \\
\hline Myalgia & $4(5.13)$ & $4(5.56)$ & 0 & 1.000 \\
\hline Headache & $3(3.85)$ & $3(4.17)$ & 0 & 1.000 \\
\hline Pharyngodynia & $7(8.97)$ & $7(9.72)$ & 0 & 1.000 \\
\hline Rhinobyon & $3(3.85)$ & $3(4.17)$ & 0 & 1.000 \\
\hline $\begin{array}{l}\text { Days from illness onset to admission, } \\
\text { median days (IQR) }\end{array}$ & $\begin{array}{l}6.5 \\
(4.0-11.25)\end{array}$ & $\begin{array}{l}6.0 \\
(4.0-11.75)\end{array}$ & $\begin{array}{l}8.5 \\
(3.0-12.25)\end{array}$ & 0.903 \\
\hline $\begin{array}{l}\text { Days from illness onset to diagnosis } \\
\text { confirmed, median days (IQR) }\end{array}$ & $5.0(3.0-8.0)$ & $5.0(3.0-7.0)$ & $\begin{array}{l}9.0 \\
(6.0-16.0)\end{array}$ & 0.042 \\
\hline $\begin{array}{l}\text { Days from diagnosis confirmed to } \\
\text { virus turned negative, median days } \\
\text { (IQR) }\end{array}$ & $\begin{array}{l}11.0 \\
(8.0-15.75)\end{array}$ & $\begin{array}{l}11.0 \\
(8.0-16.0)\end{array}$ & $\begin{array}{l}8.0 \\
(7.0-12.5)\end{array}$ & 0.240 \\
\hline
\end{tabular}


Table 2. Laboratory features of 78 COVID-19 patients.

\begin{tabular}{|c|c|c|c|c|}
\hline Characteristic & Total $(\mathrm{n}=78)$ & $\begin{array}{l}\text { Moderate }(\mathrm{n} \\
=72)\end{array}$ & Severe $(n=6)$ & $\begin{array}{l}P \\
\text { value }\end{array}$ \\
\hline $\begin{array}{l}\text { White blood cells ( } \\
\left.10^{9} / \mathrm{L}\right)\end{array}$ & $5.34(4.26-6.79)$ & $5.16(4.15-6.46)$ & 8.35 (5.73-13.15) & 0.008 \\
\hline$>10 \times 10^{9} / \mathrm{L}, \mathrm{n}(\%)$ & $6(7.69)$ & $4(5.56)$ & $2(33.33)$ & 0.065 \\
\hline Neutrophils\% & $\begin{array}{l}58.95 \\
(48.60-69.93)\end{array}$ & $\begin{array}{l}55.20 \\
(48.08-65.35)\end{array}$ & $\begin{array}{l}86.95 \\
(76.90-91.88)\end{array}$ & $\begin{array}{l}< \\
0.001\end{array}$ \\
\hline$>70$ & $18(23.08)$ & $13(18.06)$ & $5(83.33)$ & 0.002 \\
\hline $\begin{array}{l}\text { Neutrophils }(\times \\
\left.10^{9} / \mathrm{L}\right)\end{array}$ & $2.93(2.19-4.26)$ & $2.84(2.08-3.88)$ & 6.47 (5.10-11.09) & 0.001 \\
\hline$>6.3$ & $10(12.82)$ & $7(9.72)$ & $3(50.00)$ & 0.025 \\
\hline Lymphocytes\% & $\begin{array}{l}30.85 \\
(20.98-38.45)\end{array}$ & $\begin{array}{l}31.65 \\
(22.60-39.95)\end{array}$ & $9.0(4.08-13.38)$ & $\begin{array}{l}< \\
0.001\end{array}$ \\
\hline$<20$ & $17(21.79)$ & $11(15.28)$ & $6(100.00)$ & $\begin{array}{l}< \\
0.001\end{array}$ \\
\hline $\begin{array}{l}\text { Lymphocytes (× } \\
\left.10^{9} / \mathrm{L}\right)\end{array}$ & $1.52(1.12-1.85)$ & $1.57(1.18-1.89)$ & $0.74(0.42-1.31)$ & 0.002 \\
\hline$<1.0 \times 10^{9} / \mathrm{L}, \mathrm{n}(\%)$ & 14 (17.95) & $10(13.89)$ & $4(66.67)$ & 0.007 \\
\hline Platelet $\left(\times 10^{9} / \mathrm{L}\right)$ & $\begin{array}{l}234.00 \\
(192.50-302.00)\end{array}$ & $\begin{array}{l}243.75 \\
(195.00-308.75)\end{array}$ & $\begin{array}{l}185.00 \\
(79.00-230.75)\end{array}$ & 0.022 \\
\hline$<100$ & $3(3.85)$ & $1(1.39)$ & $2(33.33)$ & 0.014 \\
\hline $\begin{array}{l}\text { C-reactive protein } \\
(\mathrm{mg} / \mathrm{L})\end{array}$ & $0.87(0.50-6.63)$ & $0.54(0.50-4.06)$ & $\begin{array}{l}58.08 \\
(19.26-90.37)\end{array}$ & $\begin{array}{l}< \\
0.001\end{array}$ \\
\hline$>10$ & $13(16.67)$ & $8(11.11)$ & $5(83.33)$ & $\begin{array}{l}< \\
0.001\end{array}$ \\
\hline $\begin{array}{l}\text { Neutrophil to } \\
\text { platelet ratio }\end{array}$ & $1.28(0.91-2.07)$ & $1.24(0.88-1.67)$ & $4.64(2.40-11.44)$ & $\begin{array}{l}< \\
0.001\end{array}$ \\
\hline$>2.3$ & 17 (21.79) & $11(15.28)$ & $6(100.00)$ & $\begin{array}{l}< \\
0.001\end{array}$ \\
\hline $\begin{array}{l}\text { Platelet to } \\
\text { lymphocyte ratio }\end{array}$ & $\begin{array}{l}158.64 \\
(121.20-216.12)\end{array}$ & $\begin{array}{l}156.84 \\
(120.79-204.17)\end{array}$ & $\begin{array}{l}250.79 \\
(103.46-492.44)\end{array}$ & 0.294 \\
\hline $\begin{array}{l}\text { Neutrophil to } \\
\text { lymphocyte ratio }\end{array}$ & $1.87(1.20-3.21)$ & 1.72 (1.17-2.77) & $9.79(5.87-22.96)$ & $\begin{array}{l}< \\
0.001\end{array}$ \\
\hline$>3.9$ & 15 (19.23) & $9(12.50)$ & $6(100.00)$ & $\begin{array}{l}< \\
0.001\end{array}$ \\
\hline $\operatorname{ALT}(\mathrm{U} / \mathrm{L})$ & $\begin{array}{l}22.00 \\
(16.00-30.25)\end{array}$ & $\begin{array}{l}22.00 \\
(16.00-29.50)\end{array}$ & $\begin{array}{l}23.00 \\
(18.00-84.75)\end{array}$ & 0.329 \\
\hline AST (U/L) & $\begin{array}{l}21.50 \\
(18.00-28.00)\end{array}$ & $21(18.00-27.00)$ & 33.5 (23.75-68.00) & 0.004 \\
\hline$>40$ & $3(3.85)$ & $1(1.39)$ & $2(33.33)$ & 0.014 \\
\hline $\begin{array}{l}\text { Total bilirubin } \\
(\mu \mathrm{mol} / \mathrm{L})\end{array}$ & 10.75 (7.18-13.62) & $11.00(7.33-13.66)$ & $9.63(6.95-12.25)$ & 0.435 \\
\hline $\begin{array}{l}\text { Direct bilirubin } \\
(\mu \mathrm{mol} / \mathrm{L})\end{array}$ & $3.24(1.95-4.24)$ & $3.23(1.74-4.31)$ & $3.27(2.23-3.83)$ & 0.879 \\
\hline Albumin $(\mathrm{g} / \mathrm{L})$ & $\begin{array}{l}42.00 \\
(38.00-44.00)\end{array}$ & $\begin{array}{l}42.00 \\
(39.00-44.00)\end{array}$ & $\begin{array}{l}35.50 \\
(32.75-38.25)\end{array}$ & 0.003 \\
\hline$<40$ & $28(35.90)$ & $22(30.56)$ & $6(100.00)$ & 0.003 \\
\hline Creatinine $(\mu \mathrm{mol} / \mathrm{L})$ & $\begin{array}{l}56.50 \\
(47.00-68.00)\end{array}$ & $\begin{array}{l}56.50 \\
(47.00-68.00)\end{array}$ & $\begin{array}{l}58.50 \\
(50.25-67.25)\end{array}$ & 0.680 \\
\hline Urea (mmol/L) & $4.09(3.28-4.94)$ & $4.10(3.27-4.90)$ & $3.91(3.22-5.77)$ & 0.817 \\
\hline $\mathrm{LDH}(\mathrm{U} / \mathrm{L})$ & $\begin{array}{l}203.50 \\
(161.00-245.00)\end{array}$ & $\begin{array}{l}197.50 \\
(160.25-229.75)\end{array}$ & $\begin{array}{l}389.50 \\
(241.50-497.50)\end{array}$ & 0.002 \\
\hline$>245$ & $19(24.36)$ & $14(19.44)$ & $5(83.33)$ & 0.003 \\
\hline Glucose (mmol/L) & $5.05(4.48-5.93)$ & $4.90(4.40-5.60)$ & $6.20(5.63-11.50)$ & 0.007 \\
\hline$>6.1$ & $16(20.51)$ & $12(16.67)$ & $4(66.67)$ & 0.017 \\
\hline CK-MB (U/L) & $16(13.00-23.75)$ & $\begin{array}{l}16.50 \\
(13.00-26.00)\end{array}$ & $\begin{array}{l}15.50 \\
(11.50-17.50)\end{array}$ & 0.264 \\
\hline Sodium (mmol/L) & $\begin{array}{l}141.00 \\
(138.75-143.00)\end{array}$ & $\begin{array}{l}141.00 \\
(139.00-143.00)\end{array}$ & $\begin{array}{l}136.00 \\
(134.25-137.50)\end{array}$ & 0.001 \\
\hline$<135$ & $5(6.41)$ & $4(5.56)$ & $1(16.67)$ & 0.337 \\
\hline $\begin{array}{l}\text { Potassium } \\
(\mathrm{mmol} / \mathrm{L})\end{array}$ & $4.10(3.70-4.60)$ & $4.10(3.70-4.60)$ & $3.65(3.33-4.50)$ & 0.670 \\
\hline Calcium (mmol/L) & $\begin{array}{l}100.00 \\
(96.00-102.00)\end{array}$ & $\begin{array}{l}100.00 \\
(97.00-102.00)\end{array}$ & $\begin{array}{l}96.50 \\
(93.75-98.00)\end{array}$ & 0.015 \\
\hline$<96$ & $13(16.67)$ & $11(15.28)$ & $2(33.33)$ & 0.569 \\
\hline
\end{tabular}

Continuous variables are presented as median with interquartile range (IQR; $25 \%$, $75 \%$ ) or mean \pm standard deviation (SD), as appropriate. Other values are presented as numbers (\%). $P<0.05$ was considered statistically significant.

ALT, Alanine aminotransferase; AST, Aspartate aminotransferase; LDH, lactate dehydrogenase; CKMB, Creatine kinase-MB.

To further investigate the risk factors related with illness severity of COVID-19 pneumonia patients, we performed a case-control study at a ratio of 2:1. We selected 12 sex-, age- and underlying comorbidity-matched patients from the moderate patient group and matched them with 6 severe cases. The ages of the moderate patients were the same or \pm 2 years as matched to each severe case, as many of the moderate patients were younger. As shown in Table 3, we found no significant differences in the demography and clinical presentation between severe patients and the matched case-control severe cases. In addition, there were also no significant differences in the days from illness onset to admission, days from illness onset to diagnosis confirmed, and days from diagnosis confirmed to virus turned negative. Contrary to the moderate patients, there were significantly elevated concentrations of WBC, neutrophil percentages, neutrophil counts, CRP, and $\mathrm{LDH}$ in severe cases; while there were reduced levels of lymphocyte percentages, lymphocyte counts, NPR, NLR, albumin, and sodium in severe patients (Table 4). Table 3 and Table 4 show that the matched case-control severe cases had higher percentages of neutrophil percentages $>70 \%$, neutrophil counts $>6.3$ $\times 10^{9} / \mathrm{L}$, lymphocyte percentages $<20 \%$, NPR $>2.3$, NLR > 3.9, albumin < $40 \mathrm{~g} / \mathrm{L}$, and LDH > $245 \mathrm{U} / \mathrm{L}$ than those in the moderate cohorts.

Table 3. Comparison of clinical characteristics between severe cases and sex-, age-, and underlying disease-matched case-control moderate cases.

\begin{tabular}{|c|c|c|c|c|}
\hline Characteristics & $\begin{array}{l}\text { Total }(\mathrm{n}= \\
18)\end{array}$ & $\begin{array}{l}\text { Moderate } \\
(\mathrm{n}=12)\end{array}$ & $\begin{array}{l}\text { Severe }(\mathrm{n} \\
=6)\end{array}$ & $\begin{array}{l}P \\
\text { value }\end{array}$ \\
\hline Age (years) & $42.78 \pm 17.45$ & $47.75 \pm 4.26$ & $48.00 \pm 6.33$ & 0.974 \\
\hline Gender, male, n (\%) & $15(83.33)$ & $10(83.33)$ & $5(83.33)$ & 1.000 \\
\hline \multicolumn{5}{|l|}{ Coexisting diseases, $\mathrm{n}(\%)$} \\
\hline Hypertension & $4(22.22)$ & $3(25.00)$ & $1(16.67)$ & 1.000 \\
\hline Diabetes & $3(16.67)$ & $2(16.67)$ & $1(16.67)$ & 1.000 \\
\hline Cardiovascular disease & $1(5.56)$ & $1(8.33)$ & 0 & 1.000 \\
\hline Tumor & $1(5.56)$ & $1(8.33)$ & 0 & 1.000 \\
\hline Cerebrovascular disease & $1(5.56)$ & $1(8.33)$ & 0 & 1.000 \\
\hline Lung diseases $^{\mathrm{a}}$ & $1(5.56)$ & 0 & $1(16.67)$ & 0.333 \\
\hline Smoking, n (\%) & $2(11.11)$ & $1(8.33)$ & $1(16.67)$ & 1.000 \\
\hline Alcohol consumption, $\mathrm{n}(\%)$ & $2(11.11)$ & $1(8.33)$ & $1(16.67)$ & 1.000 \\
\hline \multicolumn{5}{|l|}{ Symptoms, n (\%) } \\
\hline Fever & $17(94.44)$ & $11(91.67)$ & $6(100.00)$ & 1.000 \\
\hline Shortness of breath & $5(27.78)$ & $2(16.67)$ & $3(50.00)$ & 0.352 \\
\hline Dry Cough & $8(44.44)$ & $5(41.67)$ & $3(50.00)$ & 1.000 \\
\hline Sputum production & $2(11.11)$ & $2(16.67)$ & 0 & 0.529 \\
\hline Gastrointestinal symptoms & $5(27.78)$ & $3(25.00)$ & $2(33.33)$ & 1.000 \\
\hline Hemoptysis & $1(5.56)$ & 0 & $1(16.67)$ & 0.333 \\
\hline Pharyngodynia & $1(5.56)$ & $1(8.33)$ & 0 & 1.000 \\
\hline $\begin{array}{l}\text { Days from illness onset to admission, } \\
\text { median days (IQR) }\end{array}$ & $6.0(4.0-9.0)$ & $\begin{array}{l}5.5 \\
(3.25-7.5)\end{array}$ & $\begin{array}{l}9.0 \\
(6.0-15.5)\end{array}$ & 0.130 \\
\hline $\begin{array}{l}\text { Days from illness onset to diagnosis } \\
\text { confirmed, median days (IQR) }\end{array}$ & $6.0(4.0-9.0)$ & $\begin{array}{l}6.0 \\
(4.0-8.5)\end{array}$ & $\begin{array}{l}9.0 \\
(6.0-16.0)\end{array}$ & 0.195 \\
\hline $\begin{array}{l}\text { Days from diagnosis confirmed to virus } \\
\text { turned negative, median days (IQR) }\end{array}$ & $\begin{array}{l}9.0 \\
(7.5-15.0)\end{array}$ & $\begin{array}{l}9.5 \\
(8.0-15.0)\end{array}$ & $\begin{array}{l}8.0 \\
(7.0-12.5)\end{array}$ & 0.506 \\
\hline
\end{tabular}

\section{Discussion}

The global number of cases of SARS-CoV-2 infection has continued to rise since the start of the outbreak. Currently, SARS-CoV-2 infection has 
resulted in more than 80,000 laboratory-confirmed cases in China, with an overall mortality of appropriately 5\% [19]. Unfortunately, despite many clinical drug trials underway, such as those for remdesivir, choloroquine, or lopinavir/ritonavir, no effective licensed drugs specifically targeting SARS-CoV-2 are currently available [20-22]. The genomic sequence of SARS-CoV-2, published in 2020, shares $82 \%$ similarity with that of SARS-CoV [4, 23]. Although similar to the severe acute respiratory syndrome (SARS) that originated in Guangdong province in 2003, SARS-CoV-2 infection can develop rapidly into respiratory failure and death [24, 25]. However, knowledge of risk factors associated with the illness severity of patients remains limited. In the present study, we summarized the epidemiological characteristics and clinical features of cases of COVID-19 in Southwest Shandong province. COVID-19 patients were divided into a moderate cohort and a severe cohort, and we identified that neutrophil percentages $>70 \%$, neutrophil counts $>6.3$ $\times 10^{9} / \mathrm{L}$, lymphocyte percentages $<20 \%$, NPR $>2.3$, NLR > 3.9, albumin < $40 \mathrm{~g} / \mathrm{L}$, and LDH $>245 \mathrm{U} / \mathrm{L}$ might be risk factors for disease severity among COVID-19 pneumonia patients.

As for systemic organ indices, our results revealed that the severe COVID-19 group presented relatively elevated WBC counts, neutrophil percentages, and neutrophil counts; high CRP levels; and decreased lymphocyte percentages, lymphocyte counts, and platelet counts. Moreover, patients in the severe cohort displayed a higher prevalence of neutrophil percentages $>70 \%$, neutrophil counts $>6.3$ $\times 10^{9} / \mathrm{L}$, lymphocyte percentages $<20 \%$, lymphocyte counts $<1.0 \times 10^{9} / \mathrm{L}$, platelet $<100 \times 10^{9} / \mathrm{L}, \mathrm{CRP}>10$ $\mathrm{mg} / \mathrm{L}$ than those in the moderate group, indicating that these parameters might be risk factors associated with COVID-19 severity in the general population. These findings are consistent with other studies that have reported abnormalities in hematological parameters including neutrophilia, lymphocytopenia, and thrombocytopenia in association with disease severity or mortality for patients with COVID-19 pneumonia [26, 27]. Elevated CRP concentrations have been reported to be connected with poor outcomes and death, and more severe cases displayed more marked elevation as compared with moderate cases [26]. These laboratory data might suggest that patients with COVID-19 have impaired hematological and cellular immune systems, as reported previously [28]. In our present analysis, neutrophil percentages $>$ $70 \%$, neutrophil counts $>6.3 \times 10^{9} / \mathrm{L}$, and lymphocyte percentages $<20 \%$ remained in the sex-, age-, and comorbid illness-matched control cases. Studies involving more patients are necessary to further consolidate our preliminary conclusions. Furthermore, the mechanisms of cellular immune system dysfunction in COVID-19 deserve further investigation.

Table 4. Laboratory features of severe cases and age-sex matched case-control moderately ill patients.

\begin{tabular}{|c|c|c|c|c|}
\hline Characteristic & Total $(\mathrm{n}=18)$ & $\begin{array}{l}\text { Moderate (n } \\
=12)\end{array}$ & Severe $(n=6)$ & $\begin{array}{l}P \\
\text { value }\end{array}$ \\
\hline $\begin{array}{l}\text { White blood cells }(\times \\
\left.10^{9} / \mathrm{L}\right)\end{array}$ & $5.97(3.97-7.45)$ & $4.83(3.37-6.39)$ & $8.35(5.73-13.15)$ & 0.010 \\
\hline$>10 \times 10^{9} / \mathrm{L}, \mathrm{n}(\%)$ & $2(11.11)$ & 0 & $2(33.33)$ & 0.098 \\
\hline Neutrophils\% & $\begin{array}{l}62.90 \\
(48.45-80.80)\end{array}$ & $\begin{array}{l}49.80 \\
(47.10-63.45)\end{array}$ & $\begin{array}{l}86.95 \\
(76.90-91.88)\end{array}$ & $\begin{array}{l}< \\
0.001\end{array}$ \\
\hline$>70$ & $6(33.33)$ & $1(8.33)$ & $5(83.33)$ & 0.004 \\
\hline $\begin{array}{l}\text { Neutrophils }(\times \\
\left.10^{9} / \mathrm{L}\right)\end{array}$ & $3.29(2.31-5.51)$ & $2.72(1.74-3.35)$ & $6.47(5.10-1.09)$ & $\begin{array}{l}< \\
0.001\end{array}$ \\
\hline$>6.3$ & $3(16.67)$ & 0 & $3(50.00)$ & 0.025 \\
\hline Lymphocytes\% & $\begin{array}{l}25.25 \\
(11.70-39.53)\end{array}$ & $\begin{array}{l}34.35 \\
(23.83-41.78)\end{array}$ & $9.00(4.08-13.38)$ & $\begin{array}{l}< \\
0.001\end{array}$ \\
\hline$<20$ & $6(33.33)$ & 0 & $6(100.00)$ & $\begin{array}{l}< \\
0.001\end{array}$ \\
\hline $\begin{array}{l}\text { Lymphocytes (× } \\
\left.10^{9} / \mathrm{L}\right)\end{array}$ & $1.28(0.77-1.61)$ & $1.52(1.12-1.87)$ & $0.74(0.42-1.31)$ & 0.013 \\
\hline$<1.0 \times 10^{9} / \mathrm{L}, \mathrm{n}(\%)$ & $6(33.33)$ & $2(16.67)$ & $4(66.67)$ & 0.107 \\
\hline Platelet $\left(\times 10^{9} / \mathrm{L}\right)$ & $\begin{array}{l}220.50 \\
(180.75-290.50)\end{array}$ & $\begin{array}{l}238.50 \\
(209.25-339.25)\end{array}$ & $\begin{array}{l}185.00 \\
(79.00-230.75)\end{array}$ & 0.053 \\
\hline$<100$ & $3(16.67)$ & $1(8.33)$ & $2(33.33)$ & 0.245 \\
\hline $\begin{array}{l}\text { C-reactive protein } \\
(\mathrm{mg} / \mathrm{L})\end{array}$ & $11.88(1.19-31.24)$ & $2.04(0.50-14.81)$ & $\begin{array}{l}58.08 \\
(19.26-90.37)\end{array}$ & 0.002 \\
\hline$>10$ & $9(50.00)$ & $4(33.33)$ & $5(83.33)$ & 0.131 \\
\hline $\begin{array}{l}\text { Neutrophil to } \\
\text { platelet ratio }\end{array}$ & $1.32(0.87-2.93)$ & $0.95(0.60-1.37)$ & $4.64(2.40-11.44)$ & $\begin{array}{l}< \\
0.001\end{array}$ \\
\hline$>2.3$ & $7(38.89)$ & $1(8.33)$ & $6(100.00)$ & $\begin{array}{l}< \\
0.001\end{array}$ \\
\hline $\begin{array}{l}\text { Platelet to } \\
\text { lymphocyte ratio }\end{array}$ & $\begin{array}{l}167.21 \\
(124.55-299.95)\end{array}$ & $\begin{array}{l}157.58 \\
(121.95-225.02)\end{array}$ & $\begin{array}{l}250.79 \\
(103.46-492.44)\end{array}$ & 0.335 \\
\hline $\begin{array}{l}\text { Neutrophil to } \\
\text { lymphocyte ratio }\end{array}$ & $2.47(1.19-6.98)$ & $1.48(1.16-2.60)$ & $9.79(5.87-22.96)$ & $\begin{array}{l}< \\
0.001\end{array}$ \\
\hline$>3.9$ & $6(33.33)$ & 0 & $6(100.00)$ & $\begin{array}{l}< \\
0.001\end{array}$ \\
\hline $\operatorname{ALT}(\mathrm{U} / \mathrm{L})$ & $\begin{array}{l}24.00 \\
(18.25-49.50)\end{array}$ & $\begin{array}{l}24.00 \\
(17.00-41.75)\end{array}$ & $\begin{array}{l}23.00 \\
(18.00-84.75)\end{array}$ & 0.892 \\
\hline AST (U/L) & $\begin{array}{l}25.50 \\
(22.00-36.00)\end{array}$ & $\begin{array}{l}24.50 \\
(21.25-29.25)\end{array}$ & $\begin{array}{l}33.50 \\
(23.75-68.00)\end{array}$ & 0.125 \\
\hline$>40$ & $3(16.67)$ & $1(8.33)$ & $2(33.33)$ & 0.245 \\
\hline $\begin{array}{l}\text { Total bilirubin } \\
(\mu \mathrm{mol} / \mathrm{L})\end{array}$ & $10.38(8.53-13.70)$ & $10.75(9.00-13.90)$ & $9.63(6.95-12.25)$ & 0.437 \\
\hline $\begin{array}{l}\text { Direct bilirubin } \\
(\mu \mathrm{mol} / \mathrm{L})\end{array}$ & $3.27(2.25-4.80)$ & $3.10(2.18-5.00)$ & $3.27(2.25-3.83)$ & 0.494 \\
\hline Albumin $(\mathrm{g} / \mathrm{L})$ & $\begin{array}{l}38.00 \\
(35.00-41.25)\end{array}$ & $\begin{array}{l}40.00 \\
(36.50-42.75)\end{array}$ & $\begin{array}{l}35.50 \\
(32.75-38.25)\end{array}$ & 0.024 \\
\hline$<40$ & $11(61.11)$ & $5(41.67)$ & $6(100.00)$ & 0.038 \\
\hline Creatinine $(\mu \mathrm{mol} / \mathrm{L})$ & $\begin{array}{l}60.50 \\
(50.50-69.50)\end{array}$ & $\begin{array}{l}60.50 \\
(51.00-74.75)\end{array}$ & $\begin{array}{l}58.50 \\
(50.25-67.25)\end{array}$ & 0.616 \\
\hline Urea $(\mathrm{mmol} / \mathrm{L})$ & $3.92(3.23-4.78)$ & $3.92(3.13-4.67)$ & $3.91(3.22-5.77)$ & 0.616 \\
\hline $\mathrm{LDH}(\mathrm{U} / \mathrm{L})$ & $\begin{array}{l}236.50 \\
(200.50-353.25)\end{array}$ & $\begin{array}{l}223.00 \\
(174.25-262.00)\end{array}$ & $\begin{array}{l}389.50 \\
(241.50-497.50)\end{array}$ & 0.041 \\
\hline$>245$ & $8(44.44)$ & $3(25.00)$ & $5(83.33)$ & 0.043 \\
\hline Glucose (mmol/L) & $5.70(4.35-9.23)$ & $4.95(4.13-8.28)$ & $6.20(5.63-11.50)$ & 0.151 \\
\hline$>6.1$ & $8(44.44)$ & $4(33.33)$ & $4(66.67)$ & 0.321 \\
\hline CK-MB (U/L) & $\begin{array}{l}16.00 \\
(11.75-19.50)\end{array}$ & $16(11.75-24.75)$ & $\begin{array}{l}15.50 \\
(11.50-17.50)\end{array}$ & 0.553 \\
\hline Sodium $(\mathrm{mmol} / \mathrm{L})$ & $\begin{array}{l}139.00 \\
(135.00-141.25)\end{array}$ & $\begin{array}{l}140.50 \\
(137.50-142.00)\end{array}$ & $\begin{array}{l}136.00 \\
(134.25-137.50)\end{array}$ & 0.032 \\
\hline$<135$ & $2(11.11)$ & $1(8.33)$ & $1(16.67)$ & 1.000 \\
\hline Potassium (mmol/L) & $4.10(3.70-4.83)$ & $4.30(3.85-4.88)$ & $3.65(3.33-4.50)$ & 0.250 \\
\hline Calcium (mmol/L) & $\begin{array}{l}97.50 \\
(95.00-100.00)\end{array}$ & $\begin{array}{l}99.00 \\
(95.25-100.75)\end{array}$ & $96.5(93.75-98.00)$ & 0.213 \\
\hline$<96$ & $5(27.78)$ & $3(25.00)$ & $2(33.33)$ & 1.000 \\
\hline
\end{tabular}


A previous study reported that infection with SARS-CoV-2 could cause the production of a range of systemic cytokines and chemokines, such as interleukin (IL)-2, IL-6, IL-7, IL-10, tumor necrosis factor- $\alpha$, monocyte chemoattractant protein- 1 and these were significantly elevated in severe patients with COVID-19 pneumonia compared with moderate cases, leading to intense systemic inflammation [29-31]. A meta-analysis recently reported that thrombocytopenia was associated with illness severity among COVID-19 patients, and a dramatic drop in platelet counts in deceased patients [32]. Interestingly, another study reported that peak platelet count during treatment, but not platelet count at baseline, was associated with a poorer prognosis; and PLR at peak platelet counts was an independent influencing factor in severe patients evaluated by multivariate analysis, thus suggesting a degree of cytokine storm due to pronounced platelet activation [33]. In contrast, we found that platelet counts were greatly reduced at admission in the severe group compared with the moderate group, while no significant difference was discovered with regard to PLR between the two groups, demonstrating that patients infected with SARS-CoV-2 might have microcirculatory dysfunction. We suggest that drugs should be administered early enough to inhibit platelet activation and prevent thrombolysis. Further data are necessary to further investigate the role of platelets in COVID-19 pneumonia.

NPR, NLR, platelet to lymphocyte ratio (PLR), and lymphocyte to monocyte ratios have been widely studied as potential predictors of systemic inflammatory responses [34, 35]. Notably, one important finding in our results was that NPR and NLR, while not PLR, were remarkably increased in severe patients as compared to either the moderate patients or the sex-, age-, and comorbid illness-matched control cases. Besides, we defined NPR at 2.3 and NLR at 3.9 using the receiver operation characteristic curve. Moreover, NPR > 2.3 and NLR > 3.9 were risk factors for severity of patients with COVID-19 pneumonia. Our results were in line with prior studies from a cohort of patients with COVID-19 indicating that NLR is a risk factor for poor clinical outcomes or mortality $[36,37]$. Therefore, these findings suggest that unbalanced immunity showing exuberant activation of the inflammatory system and inadequate response of the adaptive immune system might lead to serious illness.

In our study, we demonstrated that severe patients were susceptible to hepatic insufficiency, which is in accordance with previous reports [38-40]. The laboratory indices reflecting derangement of liver function that were associated with disease severity of
COVID-19 pneumonia were increased AST and LDH, and reduced albumin. In addition, severe cases presented internal environment disturbance, as the laboratory parameters reflecting internal environment disturbance that related with severe illness were decreased sodium and calcium, and elevated glucose levels compared with the general population. However, there were no significant differences in the levels of potassium and sodium between critical and non-critical patients as previously reported [29]. Most importantly, LDH and albumin parameters remained valid in the sex-, age-, and underlying illness-matched control data. Therefore, more attention should be urgently paid to organ protection strategies, especially liver protection, and maintenance of internal environmental balance during the course of illness in severe COVID-19 patients.

Unlike most other retrospective studies reported previously [41-44], we conducted a perspective study in order to characterize the risk factors associated with the severity of illness among COVID-19 patients. Some prospective reports have implicated several factors related with the critical disease and mortality for patients afflicted with COVID-19; however, prospective studies of disease severity are limited [45, 46]. Reported results have demonstrated that older age, comorbidities, obesity, and laboratory abnormalities including impairment of oxygen, elevated CRP concentration, higher D-dimer level, and increased serum ferritin were associated with severe illness or mortality [14-17]. Using the continued admitted patients diagnosed with COVID-19 prospectively, we have suggested that neutrophil percentages $>70 \%$, neutrophil counts $>6.3$ $\times 10^{9} / \mathrm{L}$, lymphocyte percentages $<20 \%$, NPR $>2.3$, NLR > 3.9, albumin < $40 \mathrm{~g} / \mathrm{L}$, and LDH $>245 \mathrm{U} / \mathrm{L}$ were risk factors for illness severity in COVID-19 patients. Our study also featured a case-control study at 2:1 that further validated the findings, which were different from the prospective reports. In contrast with some studies previously [14, 15], not only did we identify some factors for illness severity, but we also further provided the specific values of these indicators in COVID-19. Therefore, our study might to some extent add some knowledge to the predictors for disease severity among COVID-19 patients.

The limitations should be addressed in further research. Due to the single-center study design and limited number of cases, caution should be applied, as some of these conclusions are preliminary. In addition, another note of caution is due to the limited number of severe cases. Since there were only six patients, it is difficult to evaluate risk factors for illness severity using univariate or multivariate methods. Moreover, since the pathogen of 
SARS-CoV-2 was identified, the dynamics of viral load and antibody titer are unclear. These need to be validated in following studies.

\section{Conclusions}

In conclusion, we identified many factors, such as shortness of breath, fatigue, longer days from illness onset to diagnosis confirmed, neutrophil percentages $>70 \%$, neutrophil counts $>6.3 \times 10^{9} / \mathrm{L}$, lymphocyte percentages $<20 \%$, lymphocyte counts $<$ $1.0 \times 10^{9} / \mathrm{L}$, platelet $<100 \times 10^{9} / \mathrm{L}, \mathrm{CRP}>10 \mathrm{mg} / \mathrm{L}$, NPR $>2.3$, NLR $>3.9$, AST $>40 \mathrm{U} / \mathrm{L}$, albumin $<40$ $\mathrm{g} / \mathrm{L}, \mathrm{LDH}>245 \mathrm{U} / \mathrm{L}$, and glucose $>6.1 \mathrm{mmol} / \mathrm{L}$ related to disease severity among COVID-19 pneumonia patients. In the sex-, age-, and comorbid illness-matched case-control study, we further revealed that neutrophil percentages $>70 \%$, neutrophil counts $>6.3 \times 10^{9} / \mathrm{L}$, lymphocyte percentages $<20 \%$, NPR $>2.3$, NLR $>3.9$, albumin $<$ $40 \mathrm{~g} / \mathrm{L}$, and $\mathrm{LDH}>245 \mathrm{U} / \mathrm{L}$ remained relative risk factors for illness severity in COVID-19 pneumonia patients with similar ages and underlying diseases.

\section{Abbreviations}

AST, aspartate aminotransferase; CRP, C-reactive protein; $\mathrm{LDH}$, lactate dehydrogenase; IL, interleukin; IQR, median with interquartile range; MERS, middle east respiratory syndrome; NLR, neutrophil to lymphocyte ratio; NPR, neutrophil to platelet ratio; PLR, platelet to lymphocyte ratio; SARS, severe acute respiratory syndrome; SARS-CoV-2, severe acute respiratory syndrome coronavirus 2; $\mathrm{SD}$, standard deviations; WBC, white blood cells.

\section{Acknowledgements}

We thank the staff of the department of infectious diseases at Jining Infectious Disease Hospital, Jining City, Shandong Province, China for their assistance in screening study subjects and collecting data. All authors would like to gratefully acknowledge the patients for their involvement.

\section{Funding}

This work was supported by grants from the National Natural Science Foundation of China (grant numbers 82000090 and 82003575), PhD Research Foundation of Affiliated Hospital of Jining Medical University (grant numbers 2020-BS-003 and 2021-BS-008), and Natural National Natural Science Foundation of Jining Medical University (grant number JYP2019KJ26).

\section{Author Contributions}

L.J., F.S. and Y.S. were responsible for research design and data interpretation. N.Z., H.Z. (Hairong
Zhang), Y.T. and F.X. collected clinical and laboratory data. N.Z., A.M. and H.Z. (Hao Zhang) analyzed data and performed statistical analyses. F.S. and N.Z. were responsible for drafting the manuscript. L.J., F.S. and Y.S. supervised, revised and had primary responsibility for the final manuscript. All authors approved the final version of the manuscript.

\section{Availability of data and materials}

All data generated or analyzed during this study are included in this published article. The data that support the findings of this study are available from the corresponding author upon reasonable request.

\section{Competing Interests}

The authors have declared that no competing interest exists.

\section{References}

1. Zhu N, Zhang D, Wang W, et al. A Novel Coronavirus from Patients with Pneumonia in China, 2019. N Engl J Med. 2020; 382: 727-33.

2. Li Q, Guan X, Wu P, et al. Early Transmission Dynamics in Wuhan, China, of Novel Coronavirus-Infected Pneumonia. N Engl J Med. 2020; 382: 1199-207.

3. Yang $\mathrm{X}, \mathrm{Yu} \mathrm{Y}, \mathrm{Xu}$ J, et al. Clinical course and outcomes of critically ill patients with SARS-CoV-2 pneumonia in Wuhan, China: a single-centered, retrospective, observational study. Lancet Respir Med. 2020; 8: 475-81.

4. Lu R, Zhao X, Li J, et al Genomic characterisation and epidemiology of 2019 novel coronavirus: implications for virus origins and receptor binding. Lancet. 2020; 395: 565-74.

5. Zhou $\mathrm{P}$, Yang $\mathrm{XL}$, Wang $\mathrm{XG}$, et al. A pneumonia outbreak associated with a new coronavirus of probable bat origin. Nature. 2020; 579: 270-3.

6. [Internet] The center for Systems Science and Engineering (CSSE) at Johns Hopkins University (JHU). Coronavirus COVID-19 global cases. (https://gisanddata.maps.arcgis.com/apps/opsdashboard/index.html\#/bda 7594740fd40299423467b48e9ecf6) (accessed September 15, 2020).

7. Chen G, Wu D, Guo W, et al. Clinical and immunological features of severe and moderate coronavirus disease 2019. J Clin Invest. 2020; 130: 2620-9.

8. Wang D, Hu B, Hu C, et al. Clinical Characteristics of 138 Hospitalized Patients With 2019 Novel Coronavirus-Infected Pneumonia in Wuhan, China. JAMA. 2020; 323: 1061-9.

9. Zhou F, Yu T, Du R, et al. Clinical course and risk factors for mortality of adult inpatients with COVID-19 in Wuhan, China: a retrospective cohort study. Lancet. 2020; 395: 1054-62.

10. Chen N, Zhou M, Dong X, et al. Epidemiological and clinical characteristics of 99 cases of 2019 novel coronavirus pneumonia in Wuhan, China: a descriptive study. Lancet. 2020; 395: 507-13.

11. Feng Y, Ling Y, Bai T, et al. COVID-19 with Different Severities: A Multicenter Study of Clinical Features. Am J Respir Crit Care Med. 2020; 201: 1380-8.

12. Richardson S, Hirsch JS, Narasimhan M, et al. Presenting Characteristics, Comorbidities, and Outcomes Among 5700 Patients Hospitalized With COVID-19 in the New York City Area. JAMA. 2020; 323: 2052-9.

13. Jiang F, Deng L, Zhang L, et al. Review of the Clinical Characteristics of Coronavirus Disease 2019 (COVID-19). J Gen Intern Med. 2020; 35: 1545-9.

14. Hajifathalian K, Kumar S, Newberry C, et al. Obesity is Associated with Worse Outcomes in COVID-19: Analysis of Early Data from New York City. Obesity (Silver Spring). 2020; 28: 1606-12.

15. Gayam V, Chobufo MD, Merghani MA, et al. Clinical characteristics and predictors of mortality in African-Americans with COVID-19 from an inner-city community teaching hospital in New York. J Med Virol 2020; [Epub ahead of print].

16. Wang $\mathrm{F}$, Hou $\mathrm{H}$, Luo $\mathrm{Y}$, et al. The laboratory tests and host immunity of COVID-19 patients with different severity of illness. JCI Insight. 2020; 5: e137799.

17. Zhang JJ, Dong X, Cao YY, et al. Clinical characteristics of 140 patients infected with SARS-CoV-2 in Wuhan, China. Allergy. 2020; 75: 1730-41.

18. National Health Commission \& State Administration of Traditional Chinese Medicine. Diagnosis and Treatment Protocol for Novel Coronavirus Pneumonia (Trial Version 7). 2020.

19. [Internet] National Health Commission of the People's Republic of China. Latest on the novel coronavirus outbreak. (http://www.nhc.gov.cn/ xcs/yqtb/202006/2b5c06dd89c94d27b0cd17a2c53b8169.shtml) (accessed June 7, 2020)

20. Beigel JH, Tomashek KM, Dodd LE, et al. Remdesivir for the Treatment of Covid-19 - Final Report. N Engl J Med. 2020; 383: 1813-1826 
21. Cao B, Wang Y, Wen D, et al. A Trial of Lopinavir-Ritonavir in Adults Hospitalized with Severe Covid-19. N Engl J Med. 2020; 382: 1787-99.

22. Liu T, Luo S, Libby P, et al. Cathepsin L-selective inhibitors: A potentially promising treatment for COVID-19 patients. Pharmacol Ther. 2020; 213:107587.

23. Chan JF, Kok KH, Zhu Z, et al. Genomic characterization of the 2019 novel human-pathogenic coronavirus isolated from a patient with atypical pneumonia after visiting Wuhan. Emerg Microbes Infect. 2020; 9: 221-36.

24. de Wit E, van Doremalen N, Falzarano D, et al. SARS and MERS: recent insights into emerging coronaviruses. Nat Rev Microbiol. 2016; 14: 523-34.

25. Yang Y, Peng F, Wang R, et al. The deadly coronaviruses: The 2003 SARS pandemic and the 2020 novel coronavirus epidemic in China. J Autoimmun. 2020; 109: 102434.

26. Wu C, Chen X, Cai Y, et al. Risk Factors Associated With Acute Respiratory Distress Syndrome and Death in Patients With Coronavirus Disease 2019 Pneumonia in Wuhan, China. JAMA Intern Med. 2020; 180: 934-43.

27. Bhatraju PK, Ghassemieh BJ, Nichols M, et al. Covid-19 in Critically Ill Patients in the Seattle Region - Case Series. N Engl J Med. 2020; 382: 2012-22.

28. Guan WJ, Ni ZY, Hu Y, et al. Clinical Characteristics of Coronavirus Disease 2019 in China. N Engl J Med. 2020; 382: 1708-20.

29. Huang C, Wang Y, Li X, et al. Clinical features of patients infected with 2019 novel coronavirus in Wuhan, China. Lancet. 2020; 395: 497-506.

30. Subbarao K, Mahanty S. Respiratory Virus Infections: Understanding COVID-19. Immunity. 2020; 52: 905-9.

31. Moore JB, June $\mathrm{CH}$. Cytokine release syndrome in severe COVID-19. Science. 2020; 368: 473-4.

32. Lippi G, Plebani M, Henry BM. Thrombocytopenia is associated with severe coronavirus disease 2019 (COVID-19) infections: A meta-analysis. Clin Chim Acta. 2020; 506: 145-8.

33. Qu R, Ling Y, Zhang YH, et al. Platelet-to-lymphocyte ratio is associated with prognosis in patients with coronavirus disease-19. J Med Virol. 2020; 92: 1533-1541.

34. Ying HQ, Deng QW, He BS, et al. The prognostic value of preoperative NLR, d-NLR, PLR and LMR for predicting clinical outcome in surgical colorectal cancer patients. Med Oncol. 2014; 31: 305.

35. Mei Z, Shi L, Wang B, et al. Prognostic role of pretreatment blood neutrophil-to-lymphocyte ratio in advanced cancer survivors: A systematic review and meta-analysis of 66 cohort studies. Cancer Treat Rev. 2017; 58: 1-13.

36. Liu Y, Du X, Chen J, et al. Neutrophil-to-lymphocyte ratio as an independent risk factor for mortality in hospitalized patients with COVID-19. J Infect. 2020; 81: e6-e12.

37. Yang AP, Liu JP, Tao WQ, et al. The diagnostic and predictive role of NLR, d-NLR and PLR in COVID-19 patients. Int Immunopharmacol. 2020; 84: 106504 .

38. Wu Z, McGoogan JM. Characteristics of and Important Lessons From the Coronavirus Disease 2019 (COVID-19) Outbreak in China: Summary of a Report of 72314 Cases From the Chinese Center for Disease Control and Prevention. JAMA. 2020; 323: 1239-42.

39. Wang D, Hu B, Hu C, et al. Clinical Characteristics of 138 Hospitalized Patients With 2019 Novel Coronavirus-Infected Pneumonia in Wuhan, China. JAMA. 2020; 323: 1061-9.

40. Bangash MN, Patel J, Parekh D. COVID-19 and the liver: little cause for concern. Lancet Gastroenterol Hepatol. 2020; 5: 529-30.

41. Paranjpe I, Russak A, De Freitas JK, et al. Clinical Characteristics of Hospitalized Covid-19 Patients in New York City. medRxiv. 2020; [Epub ahead of print].

42. Goyal P, Choi JJ, Pinheiro LC, et al. Clinical Characteristics of Covid-19 in New York City. N Engl J Med. 2020; 382: 2372-4.

43. Chen T, Dai Z, Mo P, et al. Clinical Characteristics and Outcomes of Older Patients with Coronavirus Disease 2019 (COVID-19) in Wuhan, China: A Single-Centered, Retrospective Study. J Gerontol A Biol Sci Med Sci. 2020; 75: 1788-95.

44. Sun Y, Koh V, Marimuthu K, et al. Epidemiological and Clinical Predictors of COVID-19. Clin Infect Dis. 2020; 71: 786-92.

45. Petrilli CM, Jones SA, Yang J, et al. Factors associated with hospital admission and critical illness among 5279 people with coronavirus disease 2019 in New York City: prospective cohort study. BMJ. 2020; 369: m1966.

46. Du RH, Liang LR, Yang CQ, et al. Predictors of mortality for patients with COVID-19 pneumonia caused by SARS-CoV-2: a prospective cohort study. Eur Respir J. 2020; 55: 2000524 